\title{
Full characterization of a high-power semiconductor disk laser beam with simultaneous capture of optimally sized focus and farfield
}

\author{
Carl Borgentun, ${ }^{*}$ Jörgen Bengtsson, and Anders Larsson \\ Photonics Laboratory, Department of Microtechnology and Nanoscience (MC2), \\ Chalmers University of Technology, Kemivägen 9, 412 96, Göteborg, Sweden \\ *Corresponding author: carl.borgentun@chalmers.se
}

Received 11 November 2010; revised 28 January 2011; accepted 4 February 2011; posted 7 February 2011 (Doc. ID 137908); published 11 April 2011

\begin{abstract}
We report on a beam characterization method that is based on the simultaneous measurement of the focus field and the farfield, thus avoiding problems with beam fluctuations during the measurement. By using reflections from both sides of a planoconvex lens, the method implements two branches of an optical system working simultaneously. Also, by letting the planoconvex lens be antireflection treated, and by allowing for both of the reflected fields to fill large and approximately equal areas on a camera detector array, the method significantly lowers the intensity onto the detector array, thus minimizing the need for additional disturbing attenuation filters to avoid camera saturation. In the numerical retrieval of the phase distribution, based on the measured intensity distributions of the focus and farfield, iterative propagation between the two branches is performed. The phase retrieval uses the two-step algorithm for the numerical field propagation conveniently providing an arbitrary choice of sampling distance in each plane. (c) 2011 Optical Society of America

OCIS codes: $100.5070,140.3295,140.5960$.
\end{abstract}

\section{Introduction}

For an optically pumped semiconductor disk laser (OP-SDL), also referred to as a vertical external cavity surface emitting laser, a stable cavity configuration together with matching sizes of pump beam and cavity field enables essentially single-transverse mode operation even at high powers (several watts continuous output). This makes these lasers attractive as compact sources of high-power emission in a beam with near-diffraction limited properties [1-4]. For these lasers, beam characterization is an important diagnostics tool to monitor the conditions in the gain element and external cavity that influence the creation of the lasing field, and it can also be used to conveniently extract different beam quality measures. The most widely used quality measure is probably the $M^{2}$ value $[5,6]$, the beam propagation factor,

0003-6935/11/121640-10\$15.00/0

(C) 2011 Optical Society of America which relates the divergence of the beam to that of a beam with the theoretically smallest divergence, both beams having the same waist diameter. Although simple as a concept, the best method to measure the $M^{2}$ value is not obvious, and there are several pitfalls in any practical implementation [7]. From a beam characterization point of view, the $\bar{M}^{2}$ value for a nearly single-transverse mode beam can be viewed as an aggregate measure of, mainly, the wavefront deviation from the best-fit spherical wavefront. With the development of megapixel detector arrays in cameras, it has become natural to aim at a full characterization, i.e. to determine the intensity and phase distribution of the beam cross section in some plane, which then completely determines the field in all positions along the beam. The intensity distribution is of course obtained directly by the camera; the phase distribution is obtained either directly by an additional measurement with a wavefront sensor [8] or indirectly by a numerical phase retrieval base $\bar{d}$ on captured intensity distributions in two, or 
more, planes. Whether the phase distribution is determined directly or indirectly, these methods work best for nearly single-transverse mode beams. The actual field distribution of the mode can, on the other hand, be completely arbitrary, which is highly desirable for the analysis of beams generated by OP-SDLs.

The numerical phase retrieval is often performed using different modifications of the GerchbergSaxton (GS) algorithm [9], but other iterative or gradient search methods have also been used [10]. Commonly, the two planes used to retrieve the phase are just separated by free space, or possibly a transforming lens to create the farfield [11,12]. In such systems, it is common to include additional planes to facilitate the numerical search [13-15]. However, numerical phase retrieval can be used also between planes that are separated by a complex arrangement of optical components [16].

The method we report on employs the GS algorithm for phase retrieval between two branches of an optical system, where the branches are designed to simultaneously produce the focus and the farfield, respectively, with approximately equal size on the detector array of a digital camera. In this way we ensure that the captured focus and farfield originate from the same beam, even in the presence of temporal fluctuations of the laser output. Simultaneous capture of focused images and defocused images is also used in wavefront analysis systems in telescopes, although there the amount of defocus is generally small enough so that only near-focus images are produced [17]. An additional advantage of our approach is that the average intensity and spatial resolution of the two fields on the detector array are nearly equal. In the GS algorithm we use a numerical propagation method that allows for a flexible setting of the sampling distance in any plane, which simplifies the propagation between the two branches. The optical component that splits the light into its two branches is a planoconvex lens, which makes it possible for the two branches to nearly overlap in space, since no other optical components are required in the branches, except for some attenuation filters com- mon to both branches. The number of these filters, and thus their disturbing effects, are reduced by using an antireflection coating on the planoconvex lens.

\section{Principle for the Simultaneous Capture of Focus and Farfield}

\section{A. Setup Description}

The setup for the capture of the focus and farfield of the laser beam is shown in Fig. 1. A lens, L1, focuses the output beam to a waist. After the waist the beam propagates further to the second lens, L2. This lens is planoconvex with its planar surface facing the incident beam, so the beam is partly reflected at the planar surface. The lens is slightly tilted so that the reflected beam almost retraces its incident path, but with just enough separation to insert a small mirror, without blocking the beam, that redirects the reflected beam towards the camera. In this way the amount of off-axis reflection in L2 is minimized. For clarity the small mirror is not shown in Fig. 1, but instead the reflected beam is drawn to travel directly to the camera from L2. We denote this light path through the setup Branch 1. The unfolded Branch 1 from the waist to the detector array is sketched in Fig. 2. The planar surface has no focusing effect, and thus the beam propagates in free space the entire distance from the waist to the detector array of the camera. If the propagation distance is long enough, and the waist diameter is not too large, we have, to a good approximation, the farfield of the beam incident on the detector array.

Moreover, the light that propagates into L2 is partly reflected from the curved surface and constitutes Branch 2. Since the curved surface then functions as a focusing mirror with focal length $f_{r}$, this reflection will be focused, as shown in the sketch of the unfolded Branch 2 in the figure. The reflected light in Branch 2 is also directed toward the camera, by way of the same small inserted mirror as Branch 1 . Unlike Branch 1, though, its propagation angle is very sensitive to any lateral displacement of L2, indicated in Fig. 1. Therefore, it is a simple matter to

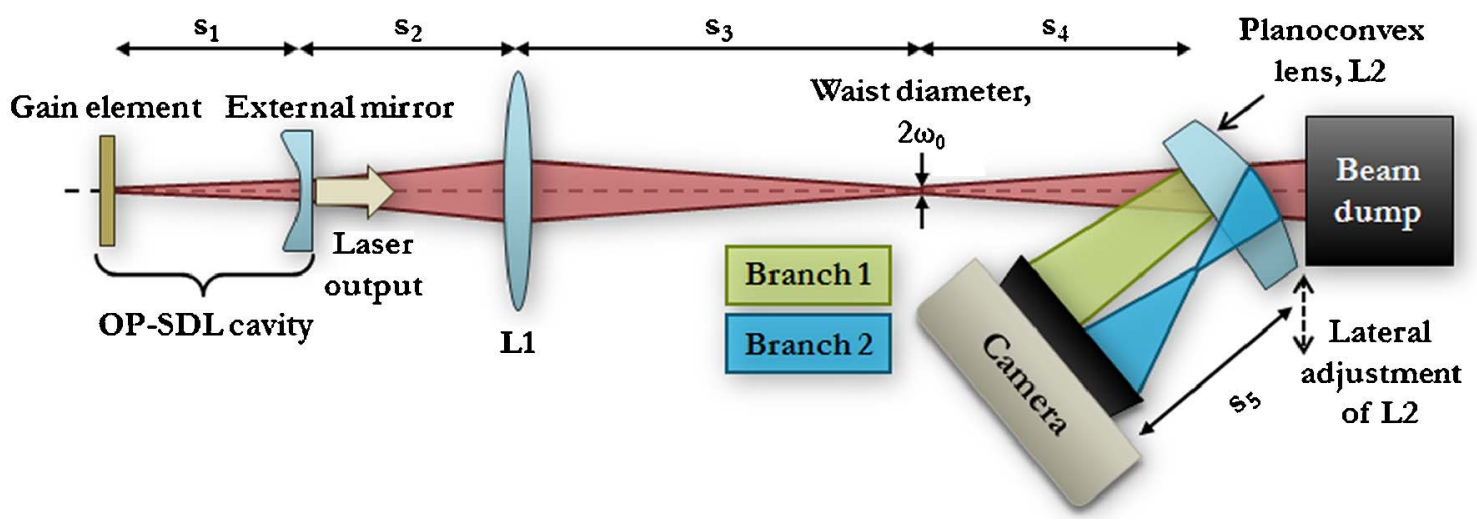

Fig. 1. (Color online) Sketch of the setup for the simultaneous capture of the focus and farfield of the beam from an OP-SDL. The tilt angle of L2 is greatly exaggerated, and the little mirror used to redirect the light reflected from L2 toward the camera is omitted for clarity. The two branches of the setup are shown unfolded in Fig. $\underline{2}$. 

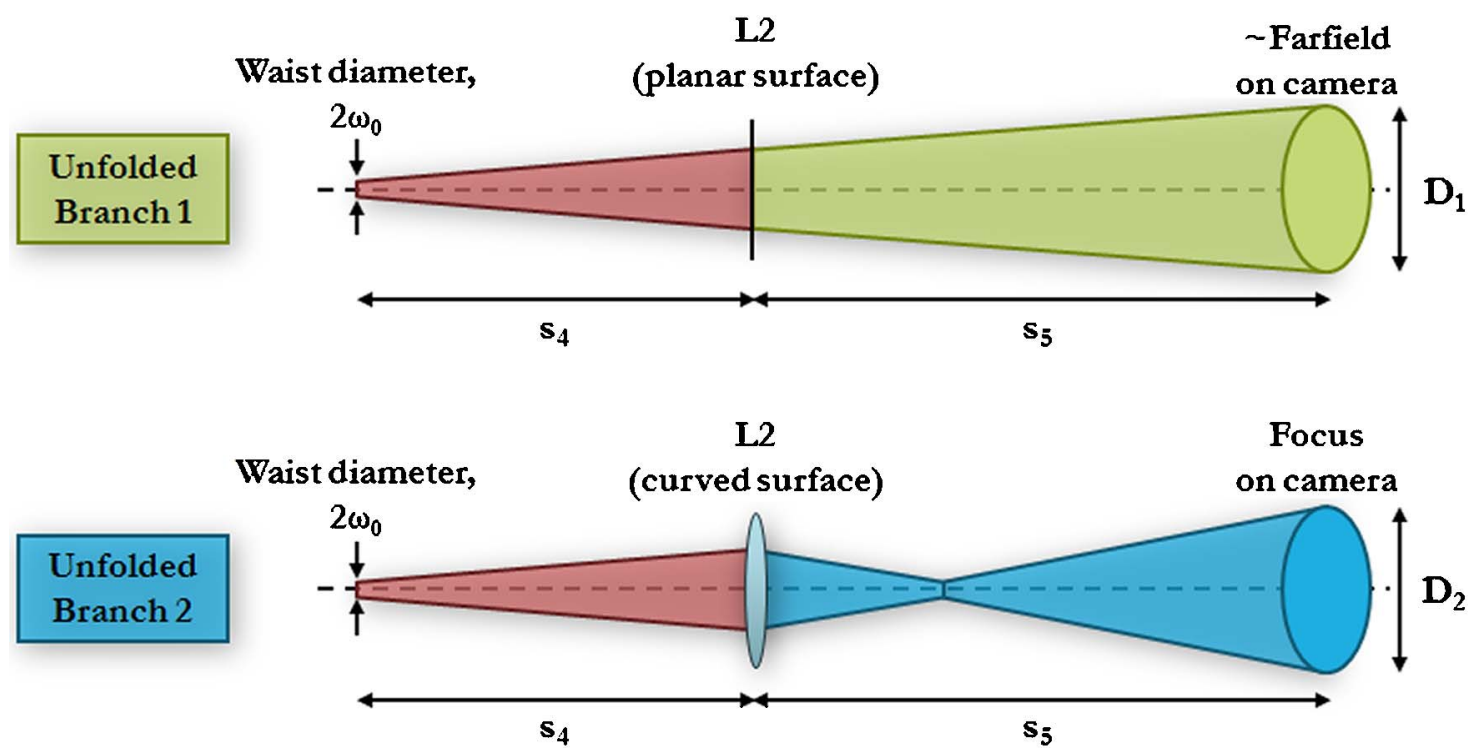

Fig. 2. (Color online) The unfolded branches 1 and 2 of the setup in Fig. 1 . The reflection in the curved surface of L2 has been drawn as equivalent to propagation through a positive lens.

center the field of Branch 2 at a different position on the detector array than the field of Branch 1 by a slight lateral movement of L2, thus allowing for simultaneous detection of both fields, provided that they do not overlap in any position. It is easy to show that $f_{r}$ is related to the specified focal length of the lens when used in transmission, $f_{2}$, by

$$
f_{r}=\frac{f_{2}\left(n_{2}-1\right)}{2 n_{2, \text { laser }}}
$$

where $n_{2}$ is the refractive index of the lens material at the wavelength for which $f_{2}$ was specified, and $n_{2 \text {,laser }}$ is the refractive index at the laser wavelength used in the beam characterization. The purpose of Branch 2 is to create an image of the waist onto the detector array, i.e. to obtain an image of the beam focus. This means that the light from a point source in any position in the plane of the waist will be focused in the camera plane. However, as indicated in Fig. 2 , for this highly coherent beam an intermediate waist is created because of the interference between the fields from all point sources. This can also be appreciated more directly by considering the significant diameter of the Gaussian beam in the waist plane. Thus, at L2 its wavefront is more planar than that of a point source, implying that the beam comes to focus at a shorter distance after L2.

\section{B. Extraction of Setup Parameters}

In addition to the requirements that Branch 1 provides the farfield and Branch 2 the beam focus, both fields should be of approximately equal size on the detector array, and that size should further be appropriate for a good fill of the array while maintaining a good separation of the two fields. To get a coarse estimation for the size of the farfield, we use the formula for the $1 / e^{2}$ diameter of a Gaussian beam on the camera array in Branch 1 [18],

$$
D_{1}=2 \omega_{0} \sqrt{1+\frac{\left(s_{4}+s_{5}\right)^{2} \lambda^{2}}{\pi^{2} \omega_{0}^{4}}},
$$

where $2 \omega_{0}$ is the beam diameter at the waist and $\lambda$ is the lasing wavelength. In Branch 2 the diameter $D_{2}$ is simply the diameter of the waist, $2 \omega_{0}$, times the magnification of the imaging system, thus

$$
D_{2}=2 \omega_{0} \frac{s_{5}}{s_{4}}
$$

Further, in order for the imaging system to actually image the plane of the waist on the plane of the detector array, the distances $s_{4}$ and $s_{5}$ must be related by the imaging condition (the lens formula)

$$
\frac{1}{f_{r}}=\frac{1}{s_{4}}+\frac{1}{s_{5}} .
$$

In Eqs. (2)-(4), the field diameters $D_{1}$ and $D_{2}$ are approximately predetermined by the requirements of filling and nonoverlap of the fields on the detector array, and $f_{r}$ is given by the available lens(es). The farfield condition for Branch 1 is not contained in these equations, but it can be checked afterwards whether $D_{1}$ is sufficiently large compared with the calculated value for $2 \omega_{0}$. Having fixed $D_{1}, D_{2}$, and $f_{r}$, we solve Eq. (2) for $\omega_{0}$ and use Eq. (4) to eliminate $s_{5}$; thus we can express $\omega_{0}$ as a function of $s_{4}$. This function is shown in Fig. 3. In this example we used $f_{2}=200 \mathrm{~mm}, n_{2}=1.51 \overline{7}, n_{2, \text { laser }}=1.508$, which yields $f_{r}=34 \mathrm{~mm}$. Further, $\lambda=990 \mathrm{~nm}$ and $D_{1}=$ $D_{2}=1.4 \mathrm{~mm}$ (the detector array size was $5 \times 7 \mathrm{~mm}$ ). In the same way, but using Eq. (3) instead of Eq. (2) to solve for $\omega_{0}$, we obtain another function for how $\omega_{0}$ must vary with $s_{4}$, shown in the same figure. As the figure shows there is a value for $\omega_{0} \approx 120 \mu \mathrm{m}$, for a distance $s_{4} \approx 40 \mathrm{~mm}$ (giving a distance $s_{5} \approx 230 \mathrm{~nm}$ ), 


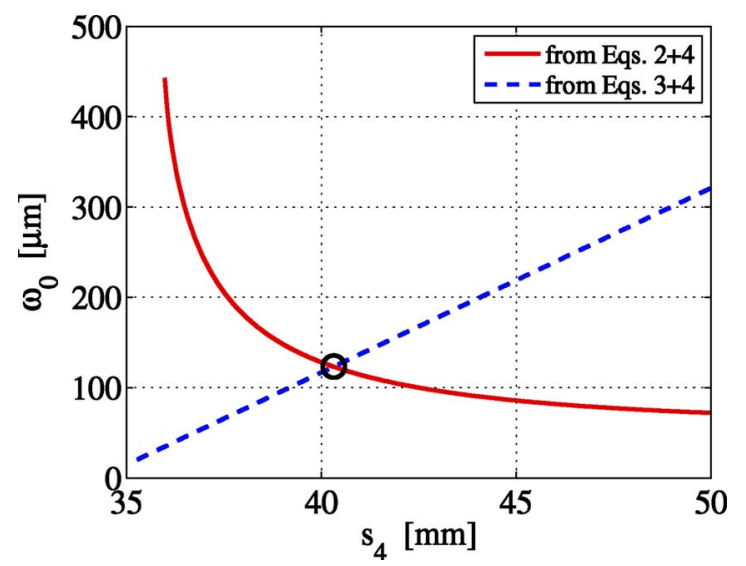

Fig. 3. (Color online) The required beam waist radius $\omega_{0}$ as a function of the distance $s_{4}$ in the setup. The intersection point indicated with a circle is the common solution to Eqs. (2)-(4) to achieve simultaneous focus and farfield capture with optimal field diameters on the camera.

for which all Eqs. (2)-(4) are fulfilled. Moreover, since $D_{1}$ is several times larger than $2 \omega_{0}$, the beam has spread considerably from the waist to the camera in Branch 1 and should thus be approaching the farfield. In the phase retrieval the actual geometry is simulated, so there is no assumption that Branch 1 should necessarily produce the farfield, but an accurate retrieval requires a significant difference between this field and the focus field. Thus, instead of using the terms focus and farfield we could have used, e.g., the terms image and direct field. This would emphasize the physical extraction of the two fields and avoid the impression that the focus and farfield are required. However, in most situations it should be desirable to use approximately these fields, and they are easily obtained as described in the next section.

We have now found a solution that fulfills all our requirements for the simultaneous capture of focus and (almost) farfield by the camera, with convenient sizes of the fields. Finally, it is straightforward to calculate the distances $s_{2}$ and $s_{3}$ to obtain a beam waist with the desired $\omega_{0}$, knowing the lens function of the external mirror of the OP-SDL and the beam size at the gain element. However, the main purpose of the calculations in this section, with the main result shown in Fig. 3, is to establish that there is indeed a solution to the problem of simultaneous capture of the optimally sized focus and farfield. In an experimental situation these values will only be used as a rough guide for how to position the components, as, for instance, the assumed beam size in the OP-SDL may deviate from the actual size. As will be described in Section $\underline{4}$, there is a simple way to experimentally adjust the system to produce approximately the focus and farfield on the camera. Once the adjustment is finished, the actual distance $s_{5}$ should be measured before being used in the phase retrieval.

\section{Phase Retrieval in the Branched System}

The phase retrieval is done numerically using the captured focus and farfield intensity distributions. Although it is not the primary purpose of this work to develop a new phase retrieval method, we will describe the algorithm we used in some detail to demonstrate phase retrieval in the branched system. The basis of the method is the GS algorithm [9] for phase retrieval. This method, and the numerous varieties thereof, consists of two operations that are repeatedly used: a numerical propagation of the optical field between the planes where the intensity has been measured, and a projection of the measured results onto the numerical, to force the numerically obtained intensities to become increasingly similar to the measured. In the branched system, the propagation must of course go back to some position that is common to both branches. To avoid unnecessary numerical propagation, we quite naturally used the last common plane: the plane immediately before the planoconvex lens.

The phase retrieval algorithm is illustrated in Fig. 4 . The processes numbered 1 and 5 are the projection operations. Originally, in the GS algorithm, this operation was simply a replacement of the numerically obtained intensity distribution in the plane with the measured distribution, while the numerically obtained phase distribution was retained. However, because of the nature of the measurement, the captured intensity values outside the actual beam, where the intensity is very low, are not reliable, since they are largely caused by stray light and/or light from the other branch. But they can significantly disturb the convergence since they extend over a large part of the captured plane. To minimize this disturbance, also the numerically obtained intensity and not only the phase is retained in the positions where the measured intensity is so low that it is not measured reliably. This idea is similar to the use of amplitude freedom in the GS design of diffractive optical elements, where it is utilized to avoid premature stagnation of the iterative algorithm and to enable particularly demanding design problems to be solved

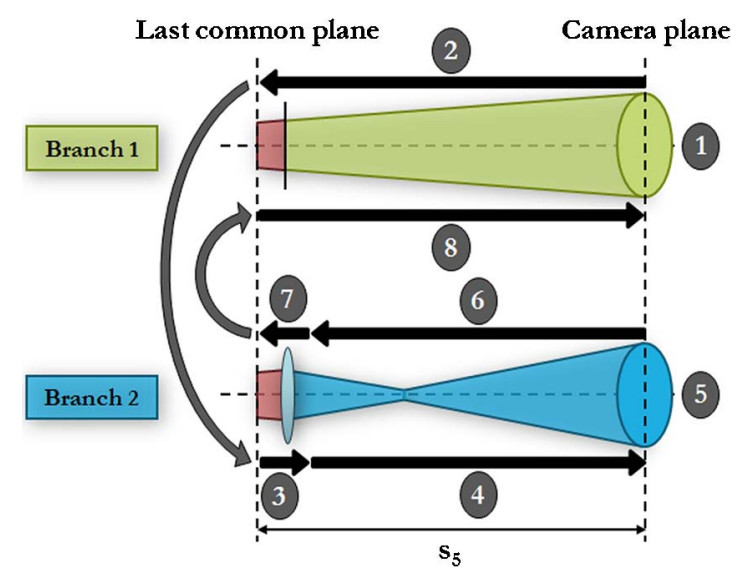

Fig. 4. (Color online) Outline of the different numerical steps in one iteration of the phase retrieval algorithm. 
$[19,20]$. In our case, one successively increases the number of those sample positions in which also the intensity is retained, until it is established that the method converges well. For instance, for our system, where no particular measures were taken to reduce stray light, we found that retaining the intensity in the positions having an intensity below $0.05 \%$ of the peak intensity in the beam cross section was sufficient for good convergence. This intensity is far lower than can reliably be measured in this type of setup, and in fact smaller than the intensity resolution in many cameras. Had this percentage been much higher, it would likely indicate that the spatial overlap of the focus and farfield in the camera plane was too large, and an adjustment of the lateral position of the planoconvex lens would be necessary to increase the field separation.

The processes numbered $2,4,6$, and 8 in the figure signify free space scalar propagation between the camera plane and the lens plane. Conventionally, this is done by using the fast Fourier transform (FFT) to either directly solve the Fresnel propagation integral or to implement the so-called propagation of angular spectrum [21]. The drawback with both these methods is that the sampling distance in the arrival plane cannot be chosen freely but is determined by the sampling distance in the starting plane (and also by the propagation distance, for the direct Fresnel integral method). For a branched system, where at least one intermediate plane is involved, a method where the arrival plane sampling distance can be more freely chosen is most convenient. In this work one such method was used: the FFT-based two-step propagation method [22], which is a formalized version of the strategy described in [16] and mathematically identical with the propagation method based on fractional Fourier transforms described in [23]. Just like the conventional methods, it can easily be modified for inverse propagation, i.e. to calculate the source field that produces a given field some distance along the direction of propagation. In our phase retrieval the fields were sampled in $512 \times$ 512 positions in each plane; the sampling distances in the camera plane and the last common plane were $4.4 \mu \mathrm{m}$ (identical with the physical pixel size of the detector array) and $1.4 \mu \mathrm{m}$, respectively.

Finally, the process denoted by 3 is simply the multiplication of the optical field by the thin-lens transmission function for a lens with focal length $f_{r}$, to simulate the propagation through the lens at the start of Branch 2. Likewise, process 7 is just the division of the same function to obtain the field just before the lens from the field just after.

In the first iteration there is no calculated field from the previous iteration, so the measured intensity distribution is used instead, together with a starting approximation for the phase distribution. We used as a starting approximation the spherical phase from a point source located at the estimated position of the beam waist, i.e. a few centimeters before the plane of the planoconvex lens. Added to this spherical phase was a stochastic phase variation with a typical lateral feature size arbitrarily chosen to be $\sim 100 \mu \mathrm{m}$ and a rms value of $\sim 1$ radian. This random phase function facilitates the extraction of phase distributions with discontinuities, as for higher order modes, which is shown in Subsection 4.B.

\section{Measurement and Evaluation Examples}

As mentioned, the calculated layout of the setup, i.e. the distances $s_{2}, \ldots, s_{5}$, is used to give an estimation of the actual positions of the optical components. For the fine-tuning, direct experimental observation is used. To do so, the setup is first adjusted so that the frontside and backside reflections from the planoconvex lens are simultaneously falling on the camera. This is readily done since the lateral adjustment of the planoconvex lens position almost solely affects the position of the light reflected from the backside. Once this is done, the longitudinal position of the focusing lens L1 is adjusted while partly obscuring the beam in the vicinity of $\mathrm{L} 1$ with a knife edge. For the farfield on the camera, there should be a corresponding partial obscuration since there is a one-to-one correspondence between each position in any two planes where the farfield is (almost) obtained, such as the knife-edge plane and the camera plane in Branch 1; see Fig. 5. The focus, and its image on the camera in Branch 2 , is related by a Fourier transform to the partly obscured field in the knife-edge plane, and hence the entire intensity distribution should be affected by the obscuration as shown in the figure, provided that L1 has been correctly positioned. The difference in the nature of the change upon obscuration between the focus image and the farfield is quite striking, so the positioning of L1 is easily done just by observing the camera image of the intensity distributions from the two branches. For clarity of illustration, in the example in the figure the OP-SDL was made to lase in a higher order mode, but the knife-edge method works for any lasing field. After this adjustment of the position of lens L1, we can be confident that the two fields on the camera are, roughly speaking, as different as possible, which is good for the accuracy of the phase retrieval.

Finally, we measure the actual distance between the camera plane and the planoconvex lens, $s_{5}$, since this distance is used in the numerical propagation processes denoted by $2,4,6$, and 8 in the previous section. Then we capture the image of the two reflections on the camera, which produces the measured intensity distributions of the focus and farfield used in steps 1 and 5 of the phase retrieval algorithm. For one example of a captured camera image, Fig. $\underline{6}$ shows the convergence behavior of the algorithm. The displayed quantity is the average phase change from one iteration to the next; more precisely it is the rms phase change in the sampling positions $(m, n)$ of the calculated field at the camera plane in Branch 1 , weighted with the calculated intensity 

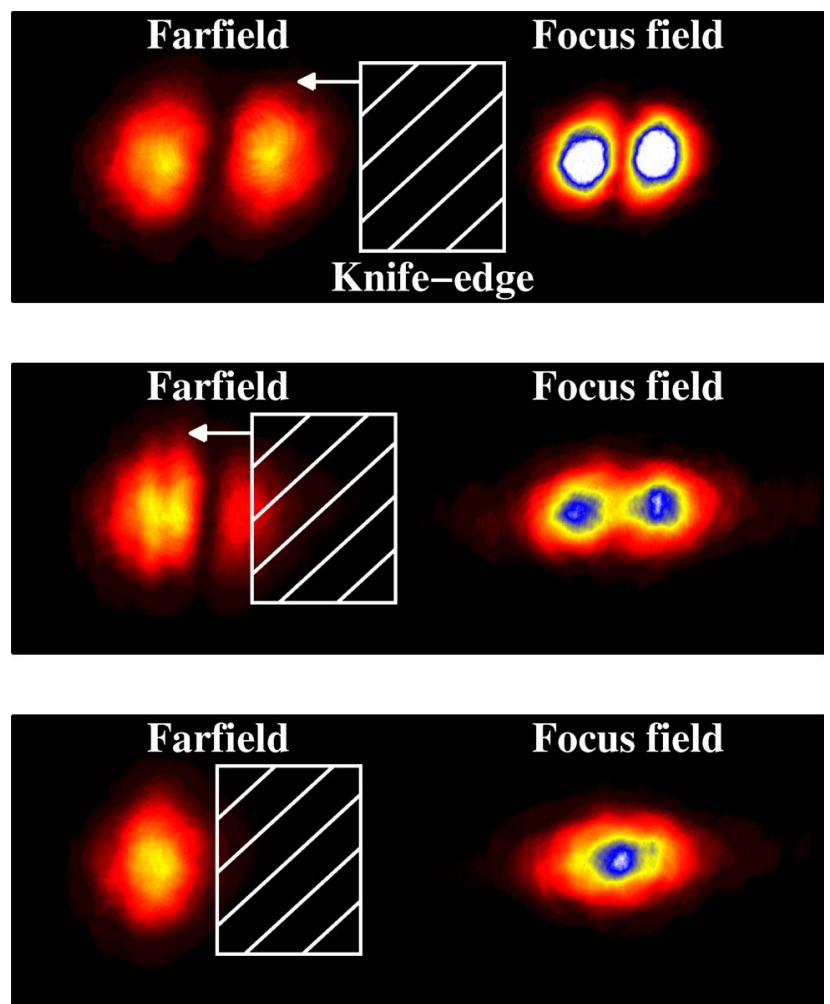

Fig. 5. (Color online) Images on the camera for the case when lens L1 has been correctly positioned. In this case the partial obscuration of the beam leads to a corresponding obscuration in the farfield image on the camera, while there is a global change in the focus image, typical for a field related to the obscured field by a Fourier transform. The OP-SDL was intentionally made to lase in a higher order mode to enhance the difference between the fields when the beam is partially obscured.

$$
\overline{\Delta \phi_{q}}=\sqrt{\frac{\sum_{m, n}\left(I_{q}(m, n)\left\{\arg \left[\frac{E_{q}(m, n)}{E_{q-1}(m, n)}\right]\right\}^{2}\right)}{\sum_{m, n} I_{q}(m, n)}}
$$

where subscript $q$ denotes results from the $q$ th iteration, and $E(m, n)$ and $I(m, n)$ are the calculated complex field and intensity distribution, respectively, in the camera plane of Branch 1 . The behavior shown in the plot is typical for GS-based methods: a robust convergence but not necessarily a monotonic decrease of the convergence parameter, in this case $\overline{\Delta \phi_{q}}$.

\section{A. Characterization and $\mathrm{M}^{2}$ Determination for a Near- Fundamental Mode Beam}

Figure 7 shows the raw image captured by the camera, from which the measured intensities used in the phase retrieval, also shown in the figure, are obtained by cutting out two appropriate portions of the captured intensity distribution. Further, the calculated intensities resulting from the phase retrieval after 100 iterations are shown (after the forward propagation from the lens plane but, of course, before the projection operation). As can be seen, the re-

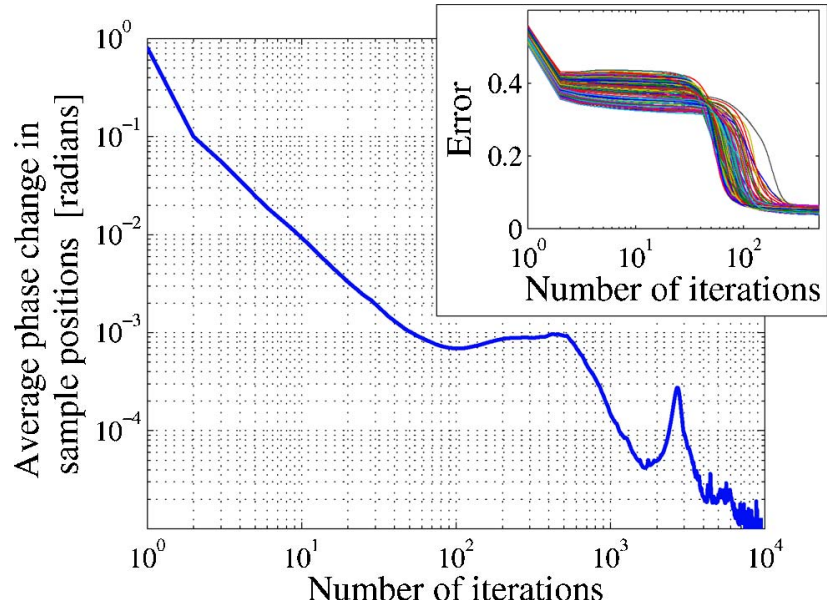

Fig. 6. (Color online) Convergence of the phase retrieval algorithm. The plotted quantity is the average phase change in a sample position from one iteration to the next, as defined in Eq. (5), for the example shown in Fig. 7. Inset, evolution of the reconstruction error, as defined in Eq. (6), with the number of iterations for the 130 trials used in the Monte Carlo simulation of the accuracy of the $M^{2}$ determination.

trieved phase manages to produce intensity distributions in the two branches that are close to the measured ones. The major differences are due to artifacts in the measured images, e.g. from interference in the attenuation filters. The closeness of the two intensity distributions can also be assessed quantitatively using a rms error that is insensitive to the arbitrary field normalization that results from the phase retrieval [24],

$$
\varepsilon=\sqrt{\frac{\sum_{m, n}\left|I_{m}(m, n)-c_{\min } I(m, n)\right|^{2}}{\sum_{m, n}\left|I_{m}(m, n)\right|^{2}}},
$$

where $I_{m}(m, n)$ is the measured intensity distribution and $c_{\min }$ is the multiplication factor that minimizes the error $\varepsilon$. Using the farfield intensities, the error was determined to be $\varepsilon=0.07$. Figure 7 also shows the phase distribution of the retrieved farfield. To better see the nonideal phase variation of the beam, the spherical starting approximation was subtracted from the displayed phase. As can be seen, the beam wavefront is nearly spherical in the central part of the beam.

To show one use of the fully characterized beam, a numerical test was performed to determine the beam propagation parameter $M^{2}$. In the test the retrieved farfield at the camera plane was further propagated numerically through a focusing lens and then in free space in small steps along the direction of propagation. In each step the beam diameter was calculated, taken as the second moment diameter in the horizontal $(x)$ direction, 


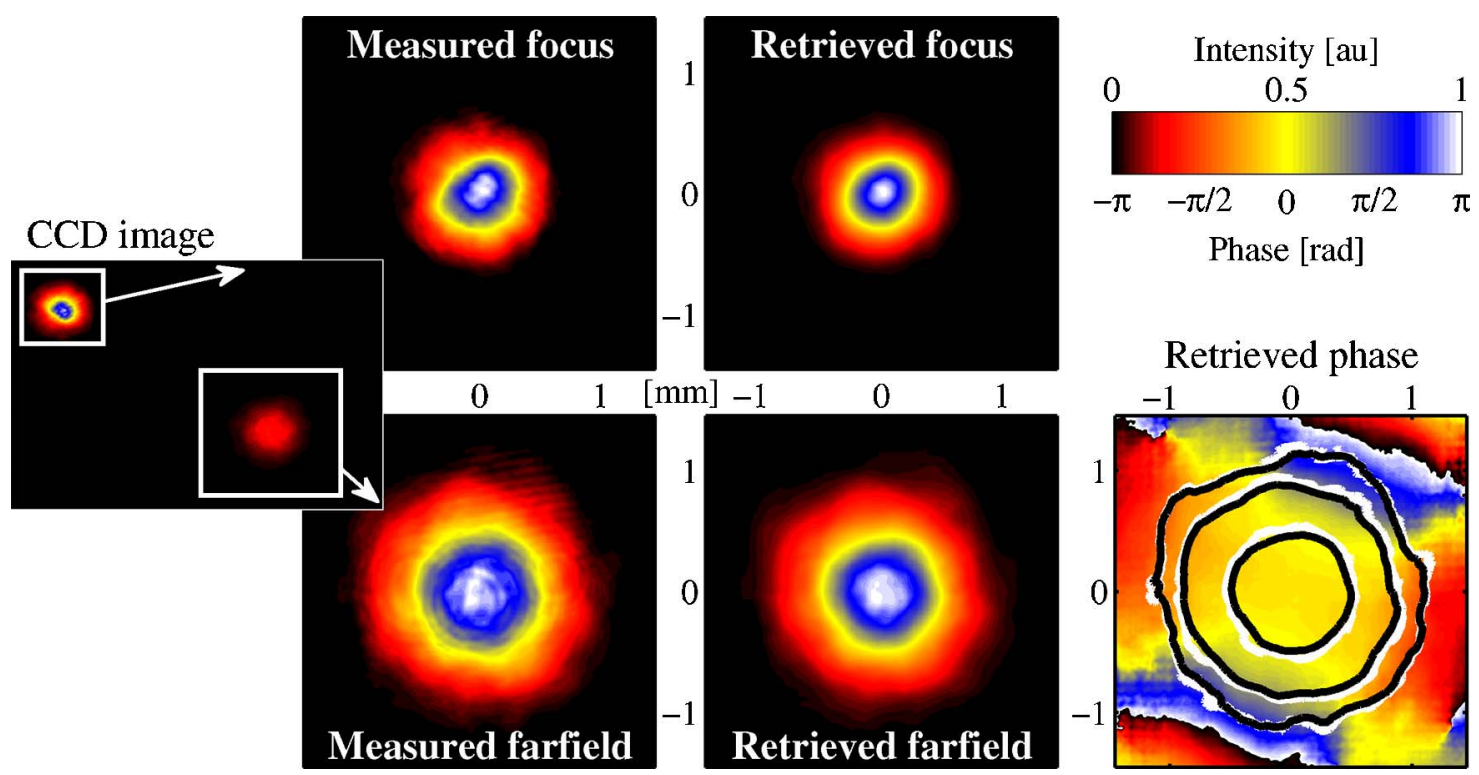

Fig. 7. (Color online) Captured camera image and the intensity distributions for the focus and farfield, together with the intensity distributions calculated with the retrieved phase distribution. The retrieved phase distribution in the farfield is also shown, where the rapidly varying spherical, deterministic part of the starting approximation has been subtracted. Overlayed are contours for the measured (white) and retrieved (black) intensity distributions for $50 \%, 10 \%$, and $2 \%$ of the peak intensity.

$$
D_{4 \sigma, x}=4 \sqrt{\frac{\sum_{m, n}\left\{I(m, n)[x(m, n)-\bar{x}]^{2}\right\}}{\sum_{m, n} I(m, n)}},
$$

where $\bar{x}$ is the center of gravity of the beam intensity distribution, and likewise in the vertical ( $y$ ) direction. The smallest obtained value for $D_{4 \sigma . x}$ was taken as the beam diameter at the waist. From the waist, the beam further propagated the long distance $L$ into the farfield, where the beam diameter $D_{4 \sigma, f f, x}$ and thus the farfield divergence $\theta_{f f, x}=D_{4 \sigma, f f, x} / L$ were calculated. The $M^{2}$ value is then obtained as [25]

$$
M_{x}^{2}=\frac{\min \left(D_{4 \sigma, x}\right) \theta_{f f, x}}{4 \lambda / \pi} .
$$

To check this value, the $M^{2}$ value was also coarsely determined by estimating the waist and farfield diameters directly from the captured camera image. Then we again use Eq. (8), but with $\min \left(D_{4 \sigma, x}\right)$ being the diameter of the captured focus field divided by the magnification of the imaging system; for $\theta_{f f, x}$ we use the diameter of the captured farfield divided by the distance $s_{4}+s_{5}$, which should be a good approximation. However, our assumption that the focus field on the camera is precisely the image of the waist is likely not quite true; rather, we image some cross section of the beam some distance away from the actual waist. Thus the value we use for the waist diameter can be significantly higher than the true value, and therefore the direct estimation should give upper bounds of the $M^{2}$ values. For the example shown in Fig. 7, the values obtained with numerical propagation of the retrieved field were $M_{x}^{2}=1.1$ and
$M_{y}^{2}=1.1$, while the coarse direct estimation gave $M_{\text {direct }, x}^{2}=1.3$ and $M_{\text {direct } y}^{2}=1.3$, which is reasonable considering the mentioned overestimation resulting from the direct method.

\section{B. Characterization and $\mathrm{M}^{2}$ Determination for a Beam with Discontinuous Phase}

To show the ability to retrieve a discontinuous phase distribution, the external mirror of the OP-SDL was slightly misaligned so that the laser output strongly resembled the Hermite-Gaussian $(1,0)$ mode, with a $\pi$-radian phase discontinuity along the zero-intensity symmetry line. For this case, Fig. 8 corresponds to Fig. 7. In the analysis the captured field was rotated to align the symmetry axes to the horizontal and vertical directions. As can be seen from the retrieved phase distribution there is indeed a $\pi$-radian phase difference between the two lobes of the field. The error in the reconstruction of the farfield intensity was lower than in the previous example, $\varepsilon=0.03$. This higher accuracy likely results from using a different area on the attenuation filters, thus avoiding the surface defect that causes the concentric disturbance that can be faintly seen in Fig. 7 in the measured farfield of the previous example. Further, the retrieved field was used for a numerical estimation of the $M^{2}$ value in the same way as described for the nearfundamental mode. The values were $M_{x}^{2}=3.3$ and $M_{y}^{2}=1.3$, while the coarse direct overestimation gave $M_{\text {direct }, x}^{2}=3.8$ and $M_{\text {direct. } y}^{2}=1.8$.

To make an estimation of the error in the obtained $M^{2}$ values, a Monte Carlo simulation of the phase retrieval was performed. In these simulations, three ingoing parameters were varied randomly with a constant probability distribution within an 


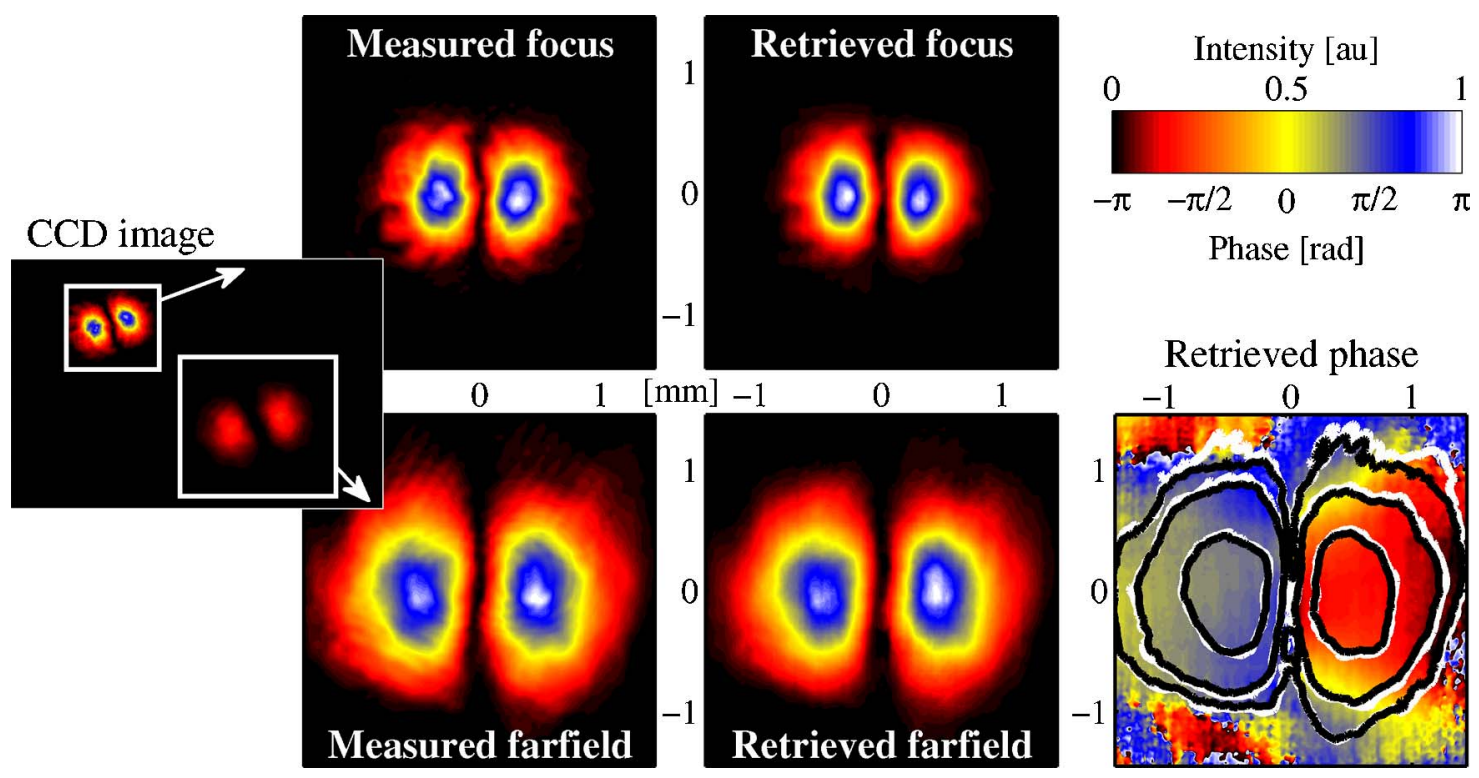

Fig. 8. (Color online) Same as Fig. 7 but for the case when the external mirror of the OP-SDL was misaligned to favor lasing on a mode with a more complex field distribution.

interval determined by assumed (fairly generous) uncertainties in the nominal/measured values: for $s_{5}$, the measured distance from lens L2 to the camera plane, this interval was $20 \mathrm{~mm}$, for the focal length of L2 it was $4 \%$ of the nominal focal length. Also we assumed a random tilt angle of L2 of up to $3^{\circ}$ from normal incidence, which to first order introduces astigmatism on the beam in Branch 2. For each set of parameter values a phase retrieval was performed. The inset in Fig. 6 shows the evolution of the reconstruction error with the number of iterations for all simulated cases; as can be seen, the initial convergence in the phase retrieval algorithm depends on the parameter values. For each retrieved phase distribution a calculation of the $M^{2}$ values followed, as described. In total, 130 such simulations were done, yielding the values $M_{x}^{2}=3.32 \pm 0.09$ and $M_{y}^{2}=$ $1.26 \pm 0.05$, where the indicated error estimation is the rms deviations from the average for these 130 sets of input parameters.

\section{Comparison with the Nominally Correct Phase Distribution}

Finally we wanted to test the method by an absolute comparison. Therefore, we deliberately introduced a known amount of astigmatism onto the laser beam to see whether this could be correctly detected. To this purpose we aligned the external mirror of the OPSDL and decreased the pump power so that the primary laser output was stable and nearly in the fundamental (Hermite-)Gaussian mode, judging from its cross-section intensity. Between the OPSDL and lens L1 a cylindrical lens with focal length $f_{c}=800 \mathrm{~mm}$ was inserted $65 \mathrm{~mm}$ before $\mathrm{L} 1$ (the distance only dictated by the space requirements for the available lens holders). The additional divergence of the beam, caused by the cylindrical lens, changed the size of the two images on the camera, so to make both images again fit on the camera the distances $s_{3}+s_{4}$ and $s_{5}$ were adjusted. This was done manually by simply observing the camera images, and the procedure required only a few seconds. The new distances were then measured and found to be $s_{3}+s_{4}=$ $390 \mathrm{~mm}$ and $s_{5}=194 \mathrm{~mm}$; the latter value was thus used in the phase retrieval algorithm. The captured CCD image is shown in Fig. 9 together with the extracted intensity distributions used in the phase retrieval, as well as the corresponding intensity distributions calculated using the retrieved phase. The reconstruction error in the far field intensity distribution was $\varepsilon=0.05$. The retrieved phase distribution is shown in Fig. 10 after subtraction of the spherically varying phase, which almost cancels the phase variation in the vertical direction. To compare the retrieved phase distribution, the optical field in Branch 1 (farfield) on the camera was simulated by a numerical propagation through the entire system, starting from the Gaussian beam at the gain element of the OP-SDL, having a planar wavefront and a width determined by the radius of curvature and position of the external mirror. This beam was successively propagated with the two-step method to the external mirror (acting as a negative lens), the inserted cylindrical lens, and L1, before it was finally propagated to the plane of the camera via the planar surface of L2. The resulting phase distribution, after subtraction of the spherical phase, is also shown in the figure, with intensity contour lines indicated. Apparently, the retrieved phase agrees remarkably well with the simulated phase, considering the nonideal laser beam and the standard lenses used.

Quantitatively, the retrieved phase front was characterized in two ways. First, the degree of astigmatism was determined by fitting the phase distribution of the central part of the beam to a 


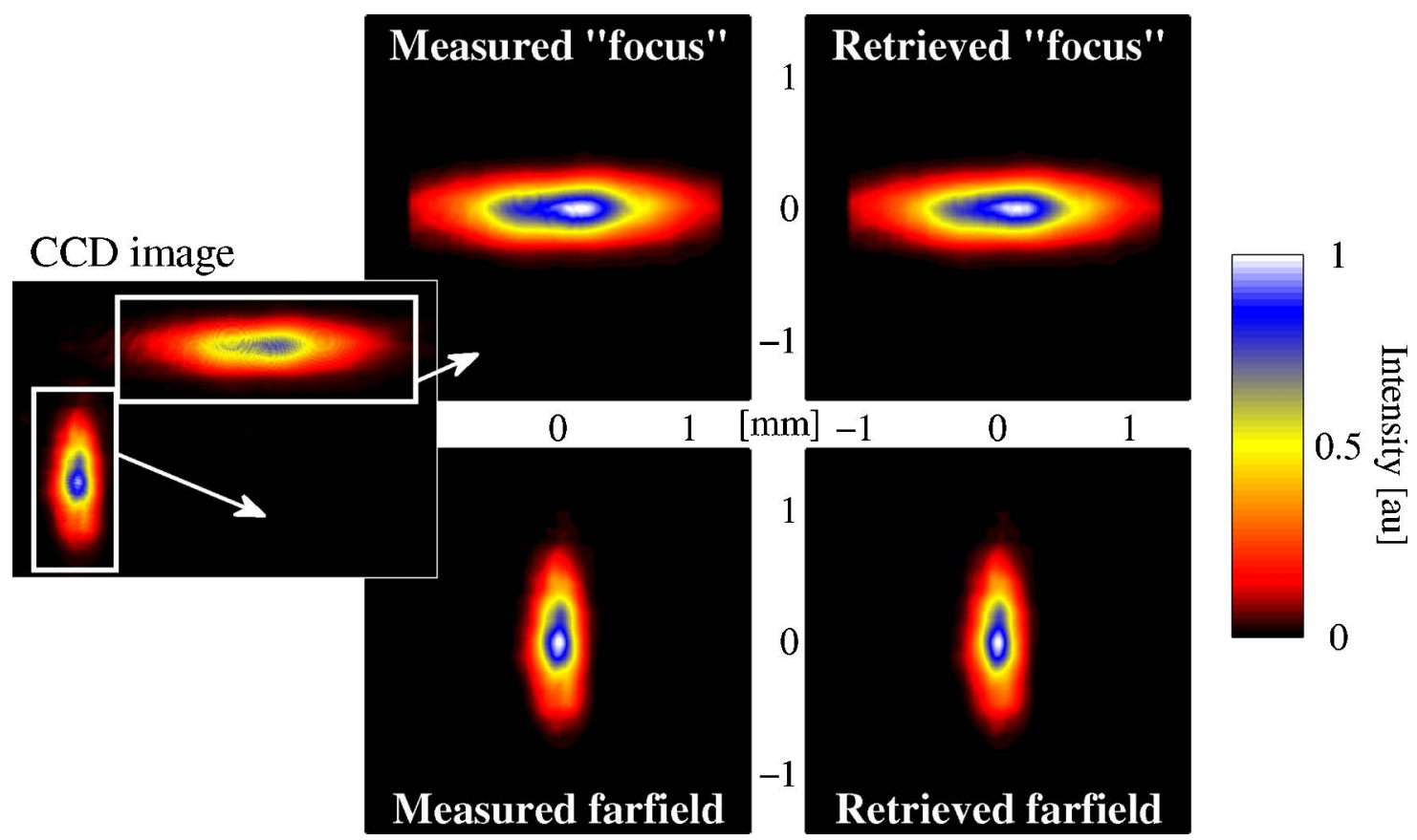

Fig. 9. (Color online) Measured and retrieved intensity distributions, as in Fig. 7 but for the case when a cylindrical lens was inserted before $\mathrm{L} 1$ to introduce astigmatism.

cylindrical wavefront. The cylindrical wavefront having the best fit to the retrieved phase, defined as the smallest intensity-weighted rms deviation of the two phase distributions, had radii of curvature in the horizontal and vertical direction of $\left.\left(R_{x}, R_{y}\right)\right|_{\text {retrieved }}=$ $(129,257) \mathrm{mm}$, which agrees to within $1 \%$ with the best fit of a cylindrical wavefront to the nominally correct phase distribution, with a curvature of $\left.\left(R_{x}, R_{y}\right)\right|_{\text {nominal }}=(129,259) \mathrm{mm}$. Second, the rms deviation of the retrieved phase from the nominally

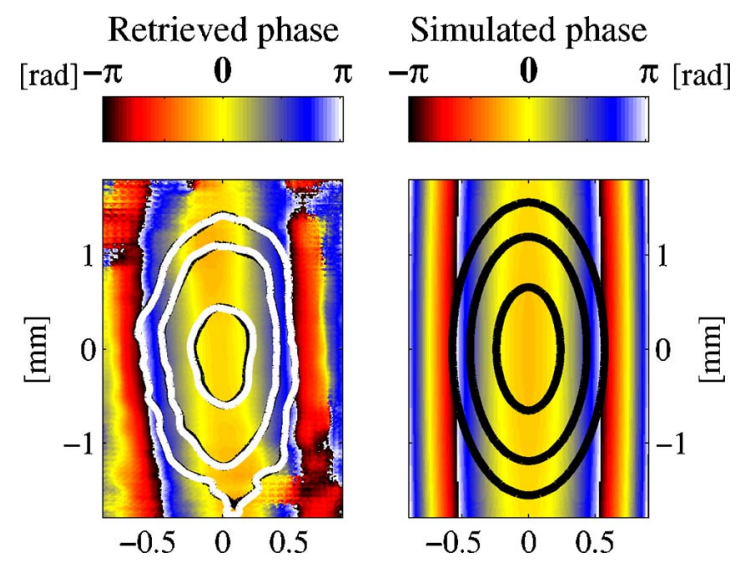

Fig. 10. (Color online) Left, retrieved phase distribution, as in Fig. 7 but for the case when a cylindrical lens was inserted before L1 to introduce astigmatism. Overlayed are contours for the measured (white) and retrieved (black, partly obscured by the white) intensity distributions for $50 \%, 10 \%$, and $2 \%$ of the peak intensity. Right, phase distribution in the farfield on the camera as calculated with a numerical propagation from the gain element of the OP-SDL through the entire optical system, assuming the field in the cavity of the OP-SDL to be an ideal Gaussian beam. Overlayed are black contour lines for the calculated intensity distribution for $50 \%, 10 \%$, and $2 \%$ of the peak intensity. correct phase was calculated. This phase deviation, again weighted with the intensity distribution, was $0.2 \mathrm{rad}$, or equivalently a $\sim \lambda / 30$ wavefront deviation, which should be more than enough accuracy for the intended type of characterization of a mediumquality high-power laser beam. If no weighting was used, but the phase deviation was calculated only in those parts of the farfield where the intensity was at least $1 \%(10 \%)$ of the peak intensity, the rms wavefront difference between the retrieved and nominally correct phase distributions was $\sim \lambda / 17(\sim \lambda / 40)$.

\section{Conclusion}

We have demonstrated that it is possible to achieve simultaneous capture of conveniently sized focus and farfield of a beam from an OP-SDL with an uncomplicated setup. Light is directed in two branches by using the reflection at either surface of a planoconvex lens, where one branch produces the farfield and the other images the beam focus. The practical realization is simplified by using standard antireflection coating on the planoconvex lens, which reduces the need for additional attenuation filters and the possibility to individually adjust the position of the focus image on the camera by a lateral displacement of the planoconvex lens. We have also implemented numerical phase retrieval for the branched system with the two-step propagation operation that is well suited for systems with many planes, thanks to the freedom in choosing the sampling distances. We believe that this method is one of the simplest ways to perform a full beam characterization that ensures that "the same" beam is being measured in both focus and farfield, which is important for e.g. external cavity lasers whose beam properties are sensitive to disturbances 
in the environment, as well as for other lasers with a tendency for a fluctuating output.

\section{References}

1. J. Sandusky and S. Brueck, "A CW external-cavity surfaceemitting laser," IEEE Photonics Technol. Lett. 8, 313-315 (1996).

2. B. Rudin, A. Rutz, M. Hoffmann, D. J. H. C. Maas, A.-R. Bellancourt, E. Gini, T. Südmeyer, and U. Keller, "Highly efficient optically pumped vertical-emitting semiconductor laser with more than $20 \mathrm{~W}$ average output power in a fundamental transverse mode," Opt. Lett. 33, 2719-2721 (2008).

3. T.-L. Wang, Y. Kaneda, J. M. Yarborough, J. Hader, J. V. Moloney, A. Chernikov, S. Chatterjee, S. W. Koch, B. Kunert, and W. Stolz, "High-power optically pumped semiconductor laser at $1040 \mathrm{~nm}$," IEEE Photonics Technol. Lett. 22, 661-663 (2010).

4. J. Chilla, Q.-Z. Shu, H. Zhou, E. Weiss, M. Reed, and L. Spinelli, "Recent advances in optically pumped semiconductor lasers," Proc. SPIE 6451, 645109 (2007).

5. A. E. Siegman, "Defining, measuring, and optimizing laser beam quality," Proc. SPIE 1868, 2 (1993).

6. P. Roth, A. Maclean, A. Kemp, S. Calvez, M. Dawson, and D. Burns, "Efficiency and beam quality analysis of a semiconductor disk laser," in Conference on Lasers and Electro-Optics / Quantum Electronics and Laser Science Conference and Photonic Applications Systems Technologies 2008 (CLEO 2008), Technical Digest (Optical Society of America, 2008), paper CWD3.

7. T. Johnston, Jr., "Beam propagation $\left(M^{2}\right)$ measurement made as easy as it gets: The four-cuts method," Appl. Opt. 37, 4840-4850 (1998).

8. J. V. Sheldakova, A. V. Kudryashov, V. Y. Zavalova, and T. Y. Cherezova, "Beam quality measurements with ShackHartmann wavefront sensor and $M^{2}$-sensor: Comparison of two methods," Proc. SPIE 6452, 645207 (2007).

9. R. Gerchberg and W. Saxton, "Practical algorithm for the determination of phase from image and diffraction plane pictures," Optik 35, 237-250 (1972).

10. J. R. Fienup, "Phase retrieval algorithms: A comparison," Appl. Opt. 21, 2758-2769 (1982).
11. S. Matsuoka and K. Yamakawa, "Wavefront reconstruction from intensity measurements using Fresnel phase retrieval method," Jpn. J. Appl. Phys. 38, L1183-L1185 (1999).

12. S. Matsuoka and K. Yamakawa, "Wave-front measurements of terawatt-class ultrashort laser pulses by the Fresnel phaseretrieval method," J. Opt. Soc. Am. B 17, 663-667 (2000).

13. A. Dooghin, N. Kundikova, and B. Zel'dovich, "Phase retrieval from laser beam intensity profiles," Opt. Commun. 91, 193-196 (1992).

14. L. Bruel, "Numerical phase retrieval from beam intensity measurements in three planes," Proc. SPIE 4932, 590-598 (2003).

15. S. Jonas, A. Kuczewski, and C. Thorn, "An improved phase retrieval algorithm for coherent beams," in Conference on Lasers and Electro-Optics (CLEO 2001), Technical Digest (Optical Society of America, 2001), paper CThL35.

16. J. R. Fienup, "Phase-retrieval algorithms for a complicated optical system,” Appl. Opt. 32, 1737-1746 (1993).

17. M. G. Lofdahl, R. L. Kendrick, A. Harwit, K. E. Mitchell, A. L. Duncan, J. H. Seldin, R. G. Paxman, and D. S. Acton, "Phase diversity experiment to measure piston misalignment on the segmented primary mirror of the Keck II telescope," Proc. SPIE 3356, 1190-1201 (1998).

18. J. T. Verdeyen, Laser Electronics, 3rd ed. (Prentice Hall, 1995).

19. F. Wyrowski, "Diffractive optical elements: Iterative calculation of quantized, blazed phase structures," J. Opt. Soc. Am. A 7, 961-969 (1990).

20. J. Liu, A. Caley, and M. Taghizadeh, "Symmetrical iterative Fourier-transform algorithm using both phase and amplitude freedoms," Opt. Commun. 267, 347-355 (2006).

21. J. W. Goodman, Introduction to Fourier Optics (McGrawHill, 1996).

22. C. Rydberg and J. Bengtsson, "Efficient numerical representation of the optical field for the propagation of partially coherent radiation with a specified spatial and temporal coherence function,” J. Opt. Soc. Am. A 23, 1616-1625 (2006).

23. X. Deng, B. Bihari, J. Gan, F. Zhao, and R. T. Chen, "Fast algorithm for chirp transforms with zooming-in ability and its applications," J. Opt. Soc. Am. A 17, 762-771 (2000).

24. J. R. Fienup, "Invariant error metrics for image reconstruction," Appl. Opt. 36, 8352-8357 (1997).

25. B. E. A. Saleh and M. C. Teich, Fundamentals of Photonics, 2nd ed. (Wiley, 2007). 